\title{
Nature of the singlet and triplet excitations mediating thermally activated delayed fluorescence
}

\author{
Y. Olivier, ${ }^{1}$ B. Yurash, ${ }^{2}$ L. Muccioli, ${ }^{3}$ G. D'Avino, ${ }^{4}$ O. Mikhnenko, ${ }^{2}$ J. C. Sancho-García, ${ }^{5}$ C. Adachi, ${ }^{6}$ \\ T.-Q. Nguyen, ${ }^{2}$ and D. Beljonne ${ }^{1}$ \\ ${ }^{1}$ Laboratory for Chemistry of Novel Materials, University of Mons, Place du Parc 20, B-7000 Mons, Belgium \\ ${ }^{2}$ Department of Chemistry and Biochemistry, Center for Polymers and Organic Solids, University of California, \\ Santa Barbara, California, USA \\ ${ }^{3}$ Dipartimento di Chimica Industriale “Toso Montanari”, Università di Bologna, Bologna, Italy \\ and Institut des Sciences Moléculaires, UMR 5255, University of Bordeaux, Talence, France \\ ${ }^{4}$ Grenoble Alpes University, CNRS, Institut Néel, F-38042 Grenoble, France \\ ${ }^{5}$ Departamento de Química Física, Universidad de Alicante, E-03080 Alicante, Spain \\ ${ }^{6}$ Center for Organic Photonics and Electronics Research (OPERA) Kyushu University 744 Motooka, Nishi, Fukuoka 819-0395, Japan
}

(Received 25 April 2017; published 27 December 2017)

\begin{abstract}
Despite significant efforts, a complete mechanistic understanding of thermally activated delayed fluorescence (TADF) materials has not yet been fully uncovered. Part of the complexity arises from the apparent dichotomy between the need for close energy resonance and for a significant spin-orbit coupling between alike charge-transfer singlet and triplet excitations. Here we show, in the case of reference carbazole derivatives, that this dichotomy can be resolved in a fully atomistic model accounting for thermal fluctuations of the molecular conformations and microscopic electronic polarization effects in amorphous films. These effects yield electronic excitations with a dynamically mixed charge-transfer and localized character, resulting in thermally averaged singlet-triplet energy differences and interconversion rates in excellent agreement with careful spectroscopic studies.
\end{abstract}

DOI: 10.1103/PhysRevMaterials.1.075602

Thermally activated delayed fluorescence (TADF) has opened a new paradigm for organic light-emitting diodes with the promise of internal quantum yields exceeding the $25 \%$ spin statistical limit while using light-element singlet emitters [1-3]. TADF relies on a triplet-to-singlet energy upconversion mechanism, also referred to as reverse intersystem crossing (RISC), for recycling the (75\%) nonemissive triplet excitons that would otherwise be lost as heat. Yet, because of the weak spin-orbit coupling ( $\left.V_{\mathrm{SOC}}\right)$ in organic conjugated compounds, this conversion can only be achieved by bringing the lowest energy singlet $\left(S_{1}\right)$ and triplet $\left(T_{1}\right)$ excitations into tight resonance [4-6]. The original chemical design strategy proposed by Adachi and colleagues for TADF emitters is based on partitioning hole and electron densities over different spatial regions of the same molecule in order to minimize the energy splitting, $\Delta E_{\mathrm{ST}}$, between $S_{1}$ and $T_{1}$ [7]. Though multiple successful efforts have been reported on the synthesis of a wide range of architectures with reduced $\Delta E_{\mathrm{ST}}$ showing TADF behavior, the nature of the electronic states involved in the RISC process and the underlying TADF mechanisms have remained elusive so far. The earlier view that singlet-triplet interconversion proceeds between pure charge-transfer triplet $\left({ }^{3} \mathrm{CT}\right)$ and singlet $\left({ }^{1} \mathrm{CT}\right)$ excited states has been recently challenged by Monkman and co-workers, who have invoked the role of a locally excited triplet state $\left({ }^{3} \mathrm{LE}\right)$ vibronically coupled to ${ }^{3} \mathrm{CT}$ in promoting RISC [8]. The most stringent argument against a pure CT-like mechanism relates to ElSayed's empirical rules for $V_{\mathrm{SOC}}$, forbidding (R)ISC between electronic states of similar nature such as $\pi-\pi^{*} \mathrm{CT}$ states [9]. RISC could also be promoted by hyperfine coupling (HFC) but recent electron paramagnetic resonance measurements showed that $\mathrm{V}_{\mathrm{SOC}}$ drives spin relaxation [10] and that HFC is only active when $\Delta E_{\mathrm{ST}}$ is lower than $1 \mathrm{~cm}^{-1}[11]$.

Here, we resolve the ambiguity about the nature of the electronic excitations mediating TADF through a combined computational and experimental study of two prototype compounds [Fig. 1(c)] [3], namely 1,2-bis(carbazol-9-yl)-4,5dicyanobenzene (2CzPN) and 1,2,3,5-tetrakis(carbazol-9-yl)4,6-dicyanobenzene (4CzIPN). Our computational approach builds on complementary techniques that account for the effect of a complex realistic environment on electronic excitations, thereby avoiding a priori assumptions on their nature. By combining molecular dynamics (MD) simulations to sample positional and conformational degrees of freedom, microelectrostatic (ME) calculations to assess electrostatic interactions with the polarizable environment $[12,13]$, and a previously validated Tamm-Dancoff density functional theory (TDA-DFT) approach to compute singlet and triplet excitation energies [14], wavefunctions, and $V_{\mathrm{SOC}}$ matrix, we show that (i) the adiabatic electronic excited states relevant for (R)ISC have a strongly mixed CT-LE character, with the amount of mixing fluctuating in time as the molecules explore the conformational space around their equilibrium structures; (ii) the instantaneous $V_{\text {SOC }}$ scales with the amount of LE admixture into $S_{1}$ and $T_{1}$ wave functions, and it is minimized for $\Delta E_{\mathrm{ST}}=0 \mathrm{eV}$, namely at the crossing between $S_{1}$ and $T_{1}$ potential energy surfaces corresponding to full $\mathrm{CT}$ state configurations; and (iii) the computed (R)ISC rates obtained by applying nonadiabatic transition theory on the basis of the configurationally averaged $V_{\mathrm{SOC}}$ and MD/ME reorganization energies are in excellent agreement with experimental data.

We start our theoretical investigations with a conformational analysis of the two molecules in their bulk amorphous phase [15]. The torsion angles of the electron-donating carbazole units with respect to the central electron-withdrawing dicyano-substituted phenylene core [Fig. 1(a)], which dictate their mutual electronic coupling, show broad distributions, typical of amorphous structures. The MD torsion angle distribution is bimodal for $2 \mathrm{CzPN}$ with peaks around $60^{\circ}$ and $120^{\circ}$, while these merge to form a broad massif centered 

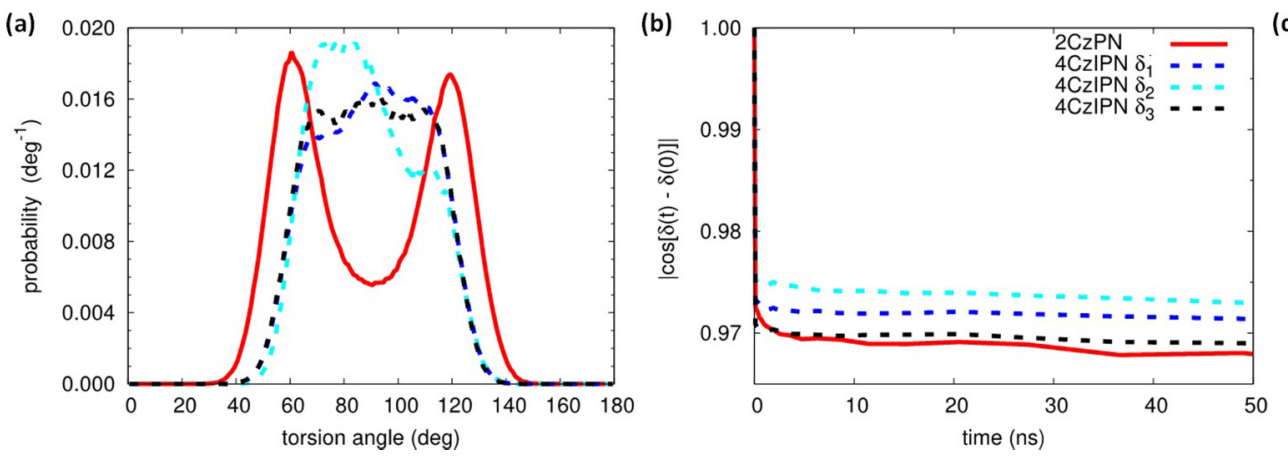

(c)

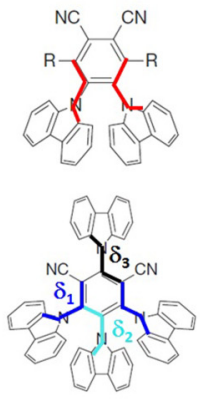

FIG. 1. (a) Torsion angle distributions for $2 \mathrm{CzPN}$ (red solid line) and for $4 \mathrm{CzIPN}\left(\delta_{1}\right.$ blue dashed line, $\delta_{2}$ cyan dashed line, $\delta_{3}$ black dashed line). (b) Autocorrelation function of the different torsion angles. (c) $2 \mathrm{CzPN}$ and $4 \mathrm{CzIPN}$ chemical structures and the color for the corresponding torsion angles.

around $90^{\circ}$ in $4 \mathrm{CzIPN}$. Thus, in $4 \mathrm{CzIPN}$ we expect on average weaker donor-acceptor interactions and, as a result, more limited CT-LE mixing compared to $2 \mathrm{CzPN}$. Importantly, the examination of the autocorrelation functions of torsion angles show two decay time scales: a short one below $1 \mathrm{~ns}$, corresponding to the oscillation of the dihedral angles around their equilibrium positions, and a long one beyond $1 \mu \mathrm{s}$ [Fig. 1(b)]. In view of the large difference between the timescales for fluctuations in dihedral angles (on the order of 10-15 degrees) and for the (R)ISC process, the molecules likely explore a large portion of the torsion potential energy surface before (R)ISC takes place. In other words, and as also suggested by Di et al. [16], in the pure solid phase of the carbazole-based molecules studied here, we expect (R)ISC to be a dynamic process gated by conformational fluctuations.

Electron and hole density plots, calculated at the DFT level upon photoexcitation, confirm segregation of the frontier molecular orbitals over the donor (D) and acceptor (A), respectively, which prefigures low-energy CT excitations [Fig. 2(a)]. To quantitatively assess the CT character in the adiabatic states, we refer to the $\phi_{S}$ index, which measures the overlap between the hole and electron densities in the attachment/detachment formalism [17]. $\phi_{S}$ evolves in space and time along with the modulation of the D-A coupling induced by the combined torsion angles around the multiple D-A single bonds and ranges from 0 in a purely (ionic)
CT-like transition to 1 for a (covalent) localized excitation $[18,19]$. TDA-DFT calculations performed on single molecule geometries extracted along the MD runs yield conformational distributions of vertical excitation energies, wave functions, and electron-hole overlaps for the bulk phase. In particular, we find a $\Delta E_{\mathrm{ST}}$ average value of $0.19 \mathrm{eV}$ in $2 \mathrm{CzPN}$, exceeding the corresponding value of $0.06 \mathrm{eV}$ in $4 \mathrm{CzIPN}$ (Table I). Out of all configurations sampled, $3 \%$ and $11 \%$ correspond to the population of molecules with $\Delta E_{\mathrm{ST}} \leqslant k_{B} T$ for $2 \mathrm{CzPN}$ and $4 \mathrm{CzIPN}$, respectively [Fig. 2(b)]. The larger $\Delta E_{\mathrm{ST}}$ in $2 \mathrm{CzPN}$ (compared to 4CzIPN) mirrors the larger difference in $\phi_{S}$ between $S_{1}$ and $T_{1}$ in that molecule; more specifically, it originates from the higher covalent character of $T_{1}$ (Table I). Not surprisingly, in both compounds the $S_{1}$ state exhibits a broader energy distribution compared to $T_{1}$ (Fig. S1 and Table S1 of the Supplemental Material [20]), a result of its larger and more dynamic CT character (as evidenced by the $\phi_{S}$ values in Table I) [21]. This arises because exchange interactions stabilize localized triplets more dramatically than their singlet counterparts, thereby prompting a more intimate LE-CT mixing in the triplet manifold. Overall, the lowest electronic excitations in the two carbazole derivatives are neither CT nor LE, but a dynamic mixture of both configurations. The smaller $\phi_{S}$ values in $4 \mathrm{CzIPN}$ compared to $2 \mathrm{CzPN}$ stem from the combined effect of the increased dihedral angles that decouple the peripheral donor moieties from the central acceptor core, (a)

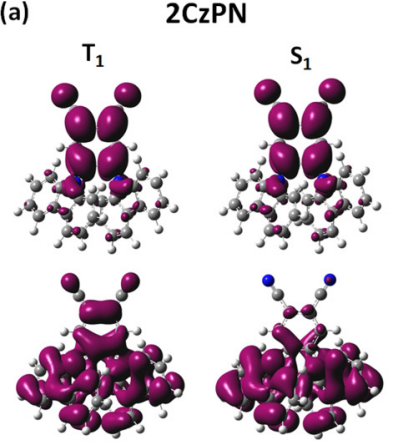

4CZIPN

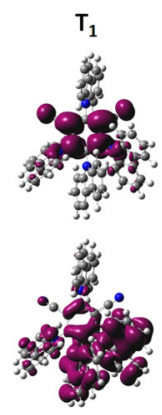

$\mathrm{S}_{1}$

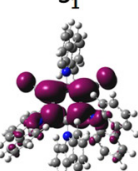

ELECTRON

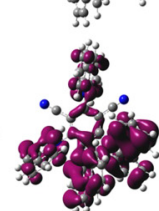

(b)

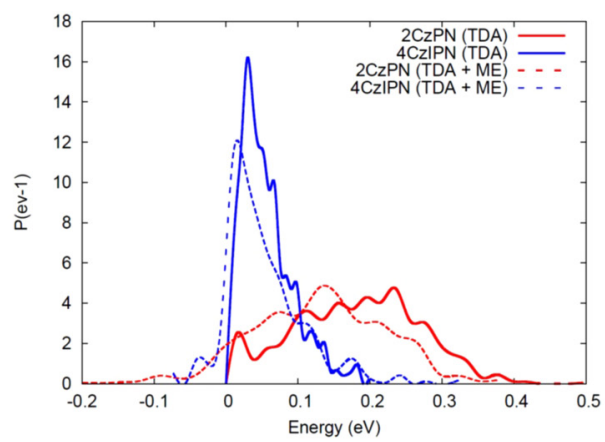

FIG. 2. (a) Hole and electron densities calculated in the attachment/detachment formalism for $T_{1}$ and $S_{1}$ excited states for $2 \mathrm{CzPN}$ and 4CzIPN. (b) Distributions of $\Delta E_{\mathrm{ST}}$ for $2 \mathrm{CzPN}$ (red) and $4 \mathrm{CzIPN}$ (blue). Solid and dashed lines correspond to results obtained with the PBE0 functional within the Tamm-Dancoff Approximation in the absence of polarizable environment and accounting for local dielectric effects, respectively. 
TABLE I. Thermally averaged singlet-triplet energy difference $\left(\Delta E_{\mathrm{ST}}\right)$, polarization energy $(P)$, overlap index $\left(\phi_{S}\right)$, and spin-orbit couplings $\left(V_{\text {SOC }}\right)$. The outer reorganization energy $\left(\lambda_{\text {out }}\right)$ is also included. All results are obtained from DFT calculations performed with the PBE0 functional and the 6-31G $(d, p)$ basis set within the Tamm-Dancoff Approximation, either in the absence of polarizable environment or accounting for local dielectric effects using a microelectrostatic (ME) scheme.

\begin{tabular}{|c|c|c|c|c|c|c|c|c|}
\hline & $\begin{array}{c}\Delta E_{\mathrm{ST}}(\mathrm{eV}) \\
\mathrm{TDA}\end{array}$ & $\begin{array}{l}\Delta E_{\mathrm{ST}}(\mathrm{eV}) \\
\mathrm{TDA}+\mathrm{ME}\end{array}$ & $\begin{array}{c}P_{T_{1}}(\mathrm{eV}) \\
\mathrm{TDA}+\mathrm{ME}\end{array}$ & $\begin{array}{c}P_{S_{1}}(\mathrm{eV}) \\
\mathrm{TDA}+\mathrm{ME}\end{array}$ & $\begin{array}{c}\phi_{S}\left(T_{1}\right) \\
\text { TDA }\end{array}$ & $\begin{array}{c}\phi_{S}\left(S_{1}\right) \\
\text { TDA }\end{array}$ & $\begin{array}{l}\lambda_{\text {out }}(\mathrm{meV}) \\
\text { TDA+ME }\end{array}$ & $\begin{array}{c}\sqrt{\left|V_{\text {SOC }}^{2}\right|(m e V)} \\
\text { TDA }\end{array}$ \\
\hline $2 \mathrm{CzPN}$ & $0.19 \pm 0.09$ & $0.14 \pm 0.09$ & $-0.20 \pm 0.11$ & $-0.25 \pm 0.12$ & $0.50 \pm 0.11$ & $0.31 \pm 0.06$ & 131 & 0.054 \\
\hline $4 \mathrm{CzIPN}$ & $0.06 \pm 0.04$ & $0.06 \pm 0.06$ & $-0.16 \pm 0.06$ & $-0.17 \pm 0.06$ & $0.31 \pm 0.07$ & $0.26 \pm 0.04$ & 31 & 0.020 \\
\hline
\end{tabular}

together with an increased hole delocalization associated with the presence of 4 , instead of 2 , carbazole units. As a matter of fact, the inverse participation ratios calculated on the basis of changes in electrostatic potential charges between the ground and excited states indicate that the hole is confined on a single carbazole unit in $2 \mathrm{CzPN}$, whereas in $4 \mathrm{CzIPN}$ it spreads over multiple carbazole (Figure S3 [20]), in line with the interpretation of pump-probe transient absorption spectroscopy data in Refs. [22,23].

Because of their partial CT character, one can anticipate that singlet and triplet excitation energies in the $2 \mathrm{CzPN}$ (and 4CzIPN) bulk are sensitive to solid-state electronic polarization effects, with a differential that directly reflects the relative magnitude of the CT contribution to their wave functions. Here, we adopt an atomistic ME scheme where excited molecules are embedded in a dielectric environment described as a set of classical point charges and anisotropic polarizabilities, accounting for both electrostatic $(\Delta S)$ and induction $(\Delta I)$ contributions. The polarization energy $(P=$ $\Delta S+\Delta I)$ quantifies the environmental contribution to $S_{1}$ and $T_{1}$ energies. The average polarization energies are computed to be $\sim-0.25 \mathrm{eV}(-0.20 \mathrm{eV})$ and $-0.17 \mathrm{eV}(-0.16 \mathrm{eV})$ for the $S_{1}\left(T_{1}\right)$ excited states of $2 \mathrm{CzPN}$ and $4 \mathrm{CzIPN}$, respectively (Table I). The broad polarization energy distributions (Figs. S4-S6 [20]) reflect the mixed CT-LE character of the excitations, with occurrences at large (small) values corresponding to high (low) CT admixture. Despite the lower CT character of $S_{1}$ and $T_{1}$ in $2 \mathrm{CzPN}$ compared to $4 \mathrm{CzIPN}$, both the electrostatic and induction components of the polarization energy are substantially larger. This results from the higher noncentrosymmetric character of the charge distribution in the former molecule, as demonstrated by the calculated excitedstate electric dipoles (Fig. S7 [20]). Because they have a similar nature, nearly equivalent electronic polarization effects are predicted for the singlet and triplet excitations in 4CzIPN, which are stabilized by about the same energy such that $\Delta E_{\mathrm{ST}}$ remains essentially unaffected with respect to the value in the absence of a polarizable environment (see Table I). The situation is different for $2 \mathrm{CzPN}$ where the more CT-like singlet excited state undergoes a larger solid-state polarization energy compared to its triplet excited state, which in turn translates into a reduced $\Delta E_{\mathrm{ST}}$ value (by $25 \%$ from 0.19 to $0.14 \mathrm{eV}$ as shown in Table I). Thus, the dielectric differential energy stabilization mechanism tends to compensate for the differences in the excited-state energies in the absence of a polarizable environment, a product of different CT-LE admixing in $S_{1}$ and $T_{1}$. The fraction of conformers that now display $\Delta E_{\mathrm{ST}}$ values lower than $k_{B} T$ rises from $3 \%(11 \%)$ in the absence of a polarizable environment to $11 \%$ (27\%) when accounting for local dielectric effects in $2 \mathrm{CzPN}$ (4CzIPN). Interestingly, some of these effects are large enough to reverse the typical ordering of excited states, resulting in negative $\Delta E_{\mathrm{ST}}$ values. Earlier works by van Voorhis and co-workers $[24,25]$ have also pointed to negative $\Delta E_{\mathrm{ST}}$ values, though this was observed for donor-acceptor intermolecular complexes and deemed to originate from enhanced kinetic exchange.

Eventually, matrix polarization effects could significantly impact $\Delta E_{\mathrm{ST}}$ and, thus, the kinetics of the whole TADF process, a strategy that has not been fully explored yet.

In TADF, spin mixing between nearly degenerate singlet and triplet states is mediated by $V_{\text {SOC. }}$. According to El-Sayed's rules, to a first-order approximation, $V_{\mathrm{SOC}}$ is expected to be vanishingly small between singlet and triplet states of the same configuration, e.g. when both $S_{1}$ and $T_{1}$ originate from $\pi-\pi *$ CT transitions [9]. In contrast, second-order nonadiabatic contributions involving higher-lying localized excitations result in non-negligible values of $V_{\text {SOC }}$ [18,26-29]. While the relevant adiabatic states in $2 \mathrm{CzPN}$ and $4 \mathrm{CzIPN}$ involve a truly mixed CT-LE character induced by vibronic coupling in both the singlet and triplet manifolds, which precludes the use of perturbation theory and requires a higher level of theory as employed above, $V_{\text {SOC }}$ is notoriously small in organic molecules and, as such, it can be treated as a perturbation. We have thus computed $V_{\text {SOC }}$ matrix elements between $S_{1}$ and $T_{1}$ by applying the zeroth-order regular approximation (ZORA) [30-32] to the full Breit-Dirac relativistic equation for a subset of 200 molecules from the MD simulations.

In line with the perturbative prescription, $V_{\mathrm{SOC}}$ turns out to be extremely small, in the range of tenths of meV. Despite these small values there is a clear correlation with $\Delta E_{\mathrm{ST}}$ [Fig. 3(a)]. In accordance with El Sayed's rules, $V_{\mathrm{SOC}}$ is vanishingly small in the case of nearly degenerate $S_{1}$ and $T_{1}$ where both excitations are mostly CT-like and thus share a similar nature (i.e., $\phi_{S}$ values). The $V_{\text {SOC }}$ surges monotonically with increasing $\Delta E_{\mathrm{ST}}$, mimicking the increased contribution of localized excitations to the wave functions. This is supported by the computed difference in $\phi_{S}$ between singlet and triplet states, i.e., $\Delta \phi_{S}=\phi_{S}\left(T_{1}\right)-\phi_{S}\left(S_{1}\right)$ [Fig. 3(b)].

At this point, one can infer that RISC in these materials results from the interplay between two opposing effects: the reduced $\Delta E_{\mathrm{ST}}$ translates into low energy barriers for the upconversion process, but it also concomitantly reduces $\mathrm{V}_{\mathrm{SOC}}$. Both magnitudes fluctuate in response to the conformationally mediated D-A interactions over time scales that are short in comparison to the (R)ISC rate. This is better apprehended by disentangling the dynamic and static contributions 
(a)

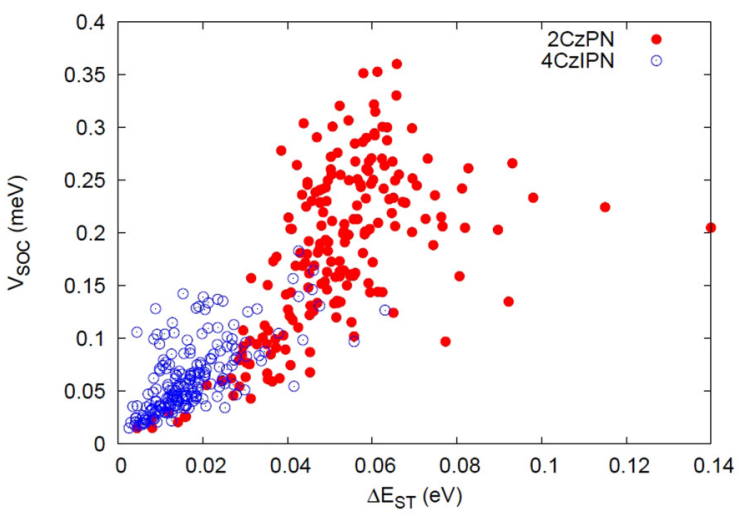

(b)

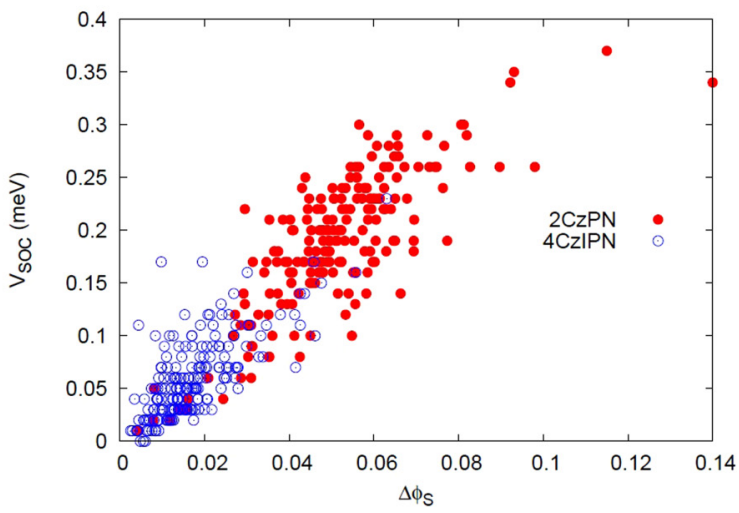

FIG. 3. Spin-orbit coupling $V_{\mathrm{SOC}}$ as a function of (a) $\Delta E_{\mathrm{ST}}$ and (b) $\Delta \phi_{S}$ in 2CzPN (blue data) and $4 \mathrm{CzIPN}$ (red data).

(see Supplemental Material [20]) to both $\Delta E_{\mathrm{ST}}$ and $V_{\mathrm{SOC}}$. In practice, the dynamic contribution is obtained from simulations where individual molecules are tracked in time over a given interval, $\tau$, and the static one by difference with respect to the full simulations (averaging over both time and space coordinates) [13]. Considering a cutoff value of 20 ns for $\tau$, we find that the dynamic component accounts for more than $80 \%$ of the $\Delta E_{\mathrm{ST}}$ variance for both $2 \mathrm{CzPN}$ and $4 \mathrm{CzIPN}$, supporting the view that the interconversion process is dynamic (Table S3 [20]). Therefore, each molecule explores its available configurational space during (R)ISC and, thus, the time-dependent $V_{\mathrm{SOC}}$ and $\Delta E_{\mathrm{ST}}$ essentially gate the spin conversion.

To properly take into account such gating effects in the calculations, it is important to probe the time evolution of the relevant electronic states as they interact with their phonon baths. Here, we directly probe $\Delta E_{\mathrm{ST}}$ and singlet/triplet wave function fluctuations along all classical nuclei degrees of freedom sampled during the MD trajectory (Figures S8 and $\mathrm{S} 9$ ). Besides providing configurationally averaged $V_{\mathrm{SOC}}$ and $\Delta E_{\mathrm{ST}}$, this approach also gives access to the outer sphere contribution to the reorganization energy, $\lambda_{\text {out }}$. In this framework, $\lambda_{\text {out }}$ for (R)ISC can indeed be evaluated as the dynamic component of the $\Delta E_{\mathrm{ST}}$ distribution accounting for the variation in $\Delta E_{\mathrm{ST}}$ due to molecular vibrations (i.e., torsional modes) and can be expressed in the classical limit as $\left(\sigma_{\Delta E_{\mathrm{ST}}}^{\mathrm{dyn}}\right)^{2}$

$\frac{\left(\sigma_{\Delta E_{\mathrm{ST}}}\right)}{2 k_{B} T}[33]$.

We evaluate the (R)ISC rates using nonadiabatic transition theory framed in the semiclassical Marcus rate expression [34]:

$$
\begin{aligned}
\left|k_{(R) \text { ISC }}\right|= & \frac{2 \pi}{\hbar}\left|V_{\text {SOC }}{ }^{2}\right| \frac{1}{\sqrt{4 \pi \lambda_{\text {out }} k_{B} T}} \\
& \times \exp \left[-\frac{\left(\lambda_{\text {out }} \pm\left|\Delta E_{\mathrm{ST}}\right|\right)^{2}}{4 \lambda_{\text {out }} k_{B} T}\right],
\end{aligned}
$$

where vertical bars denote time-averaged values and the $+(-)$ sign is associated with $k_{\mathrm{RISC}}\left(k_{\mathrm{ISC}}\right)$. The various parameters entering the rate equation together with the calculated rates are listed in Tables I and S4. Our calculations yield $\left|\Delta E_{\mathrm{ST}}\right|$ identical to the space-averaged $\Delta E_{\mathrm{ST}}$ for both compounds and $\lambda_{\text {out }}$ of 31 (121) meV in $4 \mathrm{CzIPN}$ (2CzPN). Besides showing a reduced $\Delta E_{\mathrm{ST}}, 4 \mathrm{CzIPN}$ also features a smaller $\lambda_{\text {out }}$, both results being consistent with the more similar nature of the $S_{1}$ and $T_{1}$ excited states in that molecule. Thermalized $V_{\text {SOC }}$ values for the two molecules are of the same order of magnitude, though smaller in $4 \mathrm{CzIPN}(0.017 \mathrm{meV})$ than in $2 \mathrm{CzPN}(0.054 \mathrm{meV})$, in line with the $S_{1}$ and $T_{1} \phi_{S}$ difference in $2 \mathrm{CzPN}$. Overall, the absolute values for the rates in the two molecules are mostly determined by the activation energies, with the reduced $\left|\Delta E_{\mathrm{ST}}\right|$ and $\lambda_{\text {out }}$ magnitudes in $4 \mathrm{CzIPN}$ turning into larger $\left|k_{\mathrm{RISC}}\right|$.

Before closing this theoretical section, we comment on the approximation that only classical vibrational degrees of freedom are treated explicitly in our model. In view of their similar nature, we do not expect polaronic effects induced by high-frequency quantum vibrations to significantly affect the relative energetics of the electronic states, but we cannot rule out a possible influence on the wave functions, and thus $V_{\mathrm{SOC}}$, as concluded from a very recent theoretical study [35]. In a first attempt to include such polaronic effects, we optimized the geometry of the 4CzIPN molecule in its $S_{1}$ and $T_{1}$ electronic state and used this relaxed structure for $V_{\text {SOC }}$ calculations. The results in Table S2 (see Supplemental Material [20]) indicate that polaronic effects slightly increase the CT character in the lowest adiabatic states and tend to slow down (R)ISC.

In order to corroborate the theoretical results, we also undertook an experimental investigation into 4CzIPN. (Much of the subsequent analysis could not be performed on $2 \mathrm{CzPN}$, because no ordinary biexponential decay was found, as shown in Fig. S15 [20]). The rates of ISC and RISC were assessed by comparing the photoluminescence (PL) decay of a $4 \mathrm{CzIPN}$ film with that predicted by an analytical model which describes the interplay of all of the photophysical processes that occur in TADF materials (Fig. S13 [20]) [36,37]. In this scheme, the rates of prompt $k_{p}$ and delayed fluorescence $k_{d}$ are given by

$$
k_{p, d}=\frac{1}{2}\left\{\left(k^{S}+k^{T}\right) \pm \sqrt{\left(k^{T}-k^{S}\right)^{2}+4 k_{\mathrm{ISC}} k_{\mathrm{RISC}}}\right\}
$$

where $k^{S}$ and $k^{T}$ represent the sum of the rates for decay pathways that originate in the singlet state and triplet state, respectively.

Using as initial boundary condition that only singlets are directly excited, i.e., $\left[S_{1}\right]=\left[S_{1}\right]_{0}$ and $\left[T_{1}\right]_{0}=0$ and simplifying the rate equations based on the very different 
(a)

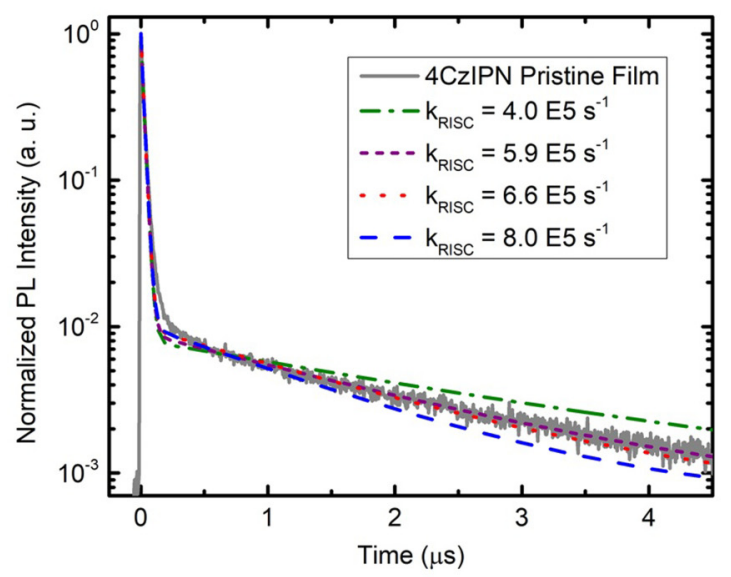

(b)

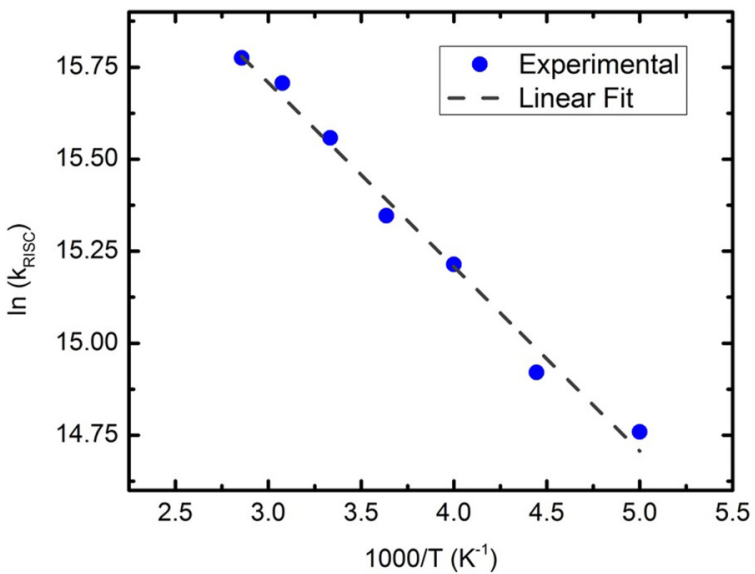

FIG. 4. (a) Calculated PL decays for neat 4CzIPN films, with $k_{\mathrm{ISC}}=2.6 \times 10^{6} \mathrm{~s}^{-1}$, compared to the experimentally measured PL decay. (b) Arrhenius plot of the rate of reverse intersystem crossing $\left(k_{\mathrm{RISC}}\right)$ in $4 \mathrm{CzIPN}$. From the slope of the linear fit, a $\Delta E_{\mathrm{ST}}$ of $42 \mathrm{meV}$ is extracted.

lifetimes of singlets and triplets, we obtain $k_{\mathrm{RISC}}$ as

$$
k_{\mathrm{RISC}}=\frac{k_{d}^{2}-k_{p} k_{d}}{k_{\mathrm{ISC}}+k_{d}-k_{p}} .
$$

Using the theoretical value corrected for polaronic effects of $2.6 \times 10^{6} \mathrm{~s}^{-1}$ for $k_{\text {ISC }}$ and the experimental values of $k_{p}$ and $k_{d}$, we obtain from Eq. (3) a value of $5.9 \times$ $10^{5} \mathrm{~s}^{-1}$ for $k_{\mathrm{RISC}}$, which is very close to the theoretical value of $6.6 \times 10^{5} \mathrm{~s}^{-1}$ (Table S4 [20]). In order to assess the validity of particular values of $k_{\mathrm{ISC}}$ and $k_{\mathrm{RISC}}$, we calculated the theoretical PL decay by using our experimental value for $k_{p}$ and the theoretical value of $k_{d}$. The calculated PL decay was then compared to the experimental PL decay, showing excellent agreement [Fig. 4(a)]. We note that the ISC and RISC rates predicted and measured here are also fully consistent with photoluminescence quenching experiments that provide an independent estimate for the spin conversion rates together with singlet and triplet exciton diffusion coefficients [38].

In order to experimentally determine $\Delta E_{\mathrm{ST}}$ in $4 \mathrm{CzIPN}$, we first estimated the temperature dependence of $k_{\mathrm{RISC}}$ using the following equation $[36,39]$ :

$$
k_{\mathrm{RISC}}(T)=\frac{k_{p}(T) k_{d}(T)}{k_{\mathrm{ISC}}} \times \frac{\Phi_{d}(T)}{\Phi_{p}(T)},
$$

where $\mathrm{k}_{\mathrm{ISC}}$ is assumed to be temperature independent (and equal to the theoretical value of $\left.2.6 \times 10^{6} \mathrm{~s}^{-1}\right)$ and $\Phi_{p}\left(\Phi_{d}\right)$ is the quantum yield of prompt (delayed) fluorescence. The method used to determine the prompt and delayed rates and quantum yields is described in the Supplemental Material [20]. Then, the natural logarithm of $k_{\mathrm{RISC}}(T)$ was plotted against the inverse temperature [Fig. 4(b)] according to the Arrhenius equation:

$$
k_{\mathrm{RISC}}(T)=\gamma \exp \left(-\frac{\Delta E_{\mathrm{ST}}}{k_{B} T}\right),
$$

where $\gamma$ is a preexponential factor. A $\Delta E_{\mathrm{ST}}$ estimate of $42 \mathrm{meV}$ was obtained for $4 \mathrm{CzIPN}$ in line with our theoretical predictions [average value of $60 \mathrm{meV}$ and most probable value of $17 \mathrm{meV}$; see Table S4 in [20] and Fig. 2(a)].
To conclude, we presented a full atomistic picture of carbazole-based TADF compounds, accounting for the interplay between conformational, positional, and electrostatic effects on their lowest singlet and triplet excited states, which quantitatively reproduces results from experimental photoluminescence studies. We showed that the electronic states involved in (R)ISC comprise a mixture of localized and charge-transfer contributions that vary with chemical structure and dynamically evolve following the changes in the molecular conformation and local dielectric environment. Indeed these dynamical effects allow meeting the two apparently incompatible conditions for an efficient TADF: a large CT component to reduce $\Delta E_{\mathrm{ST}}$ and a significant localized component to prompt spin-orbit coupling. Besides the already very vivid interest for new generations of TADF molecular emitters, this study opens up new possibilities in terms of the design of material hosts, as the intimate host-guest interactions will ultimately dictate the amplitude and dynamics of both conformational and electrostatic effects and hence triplet-to-singlet conversion rates.

The work in Mons was supported by the Programme d'Excellence de la Région Wallonne (OPTI2MAT project), the European Union's Horizon 2020 research and innovation program under Grant Agreement No. 646176 (EXTMOS project), and FNRS-FRFC. Computational resources were provided by the Consortium des Équipements de Calcul Intensif (CÉCI), funded by the Fonds de la Recherche Scientifiques de Belgique (F.R.S.-FNRS) under Grant No. 2.5020.11, as well as the Tier-1 supercomputer of the Fédération WallonieBruxelles, infrastructure funded by the Walloon Region under Grant Agreement No. 1117545. B.Y. and T.Q.N. thank the Department of the Navy, Office of Naval Research (Award No. N00014-14-1-0580) for support. L.M. acknowledges funding by the French national grant ANR-10-LABX-0042AMADEus managed by the National Research Agency under the initiative of excellence IdEx Bordeaux program (reference ANR-10-IDEX-0003-02). G.D. acknowledges support from EU through the FP7-PEOPLE-2013-IEF program (Project No. 625198). 
[1] P. F. Jones and A. R. Calloway, Chem. Phys. Lett. 10, 438 (1971).

[2] S. A. Carlsodb and D. M. Hercules, J. Am. Chem. Soc. 93, 5611 (1971).

[3] H. Uoyama, K. Goushi, K. Shizu, H. Nomura, and C. Adachi, Nature (London) 492, 234 (2012).

[4] B. S. Kim and J. Y. Lee, Adv. Funct. Mater. 24, 3970 (2014).

[5] S. Hirata, Y. Sakai, K. Masui, H. Tanaka, S. Y. Lee, H. Nomura, N. Nakamura, M. Yasumatsu, H. Nakanotani, Q. Zhang, K. Shizu, H. Miyazaki, and C. Adachi, Nat. Mater. 14, 330 (2015).

[6] J. Li, Q. Zhang, H. Nomura, H. Miyazaki, and C. Adachi, Appl. Phys. Lett. 105, 013301 (2014).

[7] K. Sato, K. Shizu, K. Yoshimura, A. Kawada, H. Miyazaki, and C. Adachi, Phys. Rev. Lett. 110, 247401 (2013).

[8] M. K. Etherington, J. Gibson, H. F. Higginbotham, T. J. Penfold, and A. P. Monkman, Nat. Commun. 7, 13680 (2016).

[9] S. Lower and M. El-Sayed, Chem. Rev. 66, 199 (1966).

[10] S. Schott, E. R. McNellis, C. B. Nielsen, H.-Y. Chen, S. Watanabe, H. Tanaka, I. McCulloch, K. Takimiya, J. Sinova, and H. Sirringhaus, Nat. Commun. 8, 15200 (2017).

[11] T. Ogiwara, Y. Wakikawa, and T. Ikoma, J. Phys. Chem. A 119, 3415 (2015).

[12] G. D’Avino, L. Muccioli, C. Zannoni, D. Beljonne, and Z. G. Soos, J. Chem. Theory Comput. 10, 4959 (2014).

[13] G. D'Avino, L. Muccioli, F. Castet, C. Poelking, D. Andrienko, Z. G. Soos, J. Cornil, and D. Beljonne, J. Phys.: Condens. Matter 28, 433002 (2016).

[14] M. Moral, L. Muccioli, W. J. Son, Y. Olivier, and J. C. SanchoGarcía, J. Chem. Theory Comput. 11, 168 (2015).

[15] M. Moral, W. J. Son, J. C. Sancho-García, Y. Olivier, and L. Muccioli, J. Chem. Theory Comput. 11, 3383 (2015).

[16] D. Di, A. S. Romanov, L. Yang, J. M. Richter, J. P. H. Rivett, S. Jones, T. H. Thomas, M. Abdi Jalebi, R. H. Friend, M. Linnolahti, M. Bochmann, and D. Credgington, Science 356, 159 (2017).

[17] A. Dreuw and M. Head-Gordon, Chem. Rev. 105, 4009 (2005).

[18] Y. Olivier, M. Moral, L. Muccioli, and J.-C. Sancho-García, J. Mater. Chem. C 5, 5718 (2017).

[19] T. Etienne, X. Assfeld, and A. Monari, J. Chem. Theory Comput. 10, 3906 (2014).
[20] See Supplemental Material at http://link.aps.org/supplemental/ 10.1103/PhysRevMaterials.1.075602 for computational, materials and experimental details.

[21] A. Köhler and D. Beljonne, Adv. Funct. Mater. 14, 11 (2004).

[22] T. Hosokai, H. Matsuzaki, A. Furube, K. Tokumaru, T. Tsutsui, H. Nakanotani, M. Yahiro, and C. Adachi, Proc. SPIE 9941, 994107 (2016).

[23] T. Hosokai, H. Matsuzaki, A. Furube, K. Tokumaru, T. Tsutsui, H. Nakanotani, M. Yahiro, and C. Adachi, SID Symp. Digest Technical Pap. 47, 786 (2016).

[24] M. Segal, M. Singh, K. Rivoire, S. Difley, T. Van Voorhis, and M. A. Baldo, Nat. Mater. 6, 374 (2007).

[25] S. Difley, D. Beljonne, and T. Van Voorhis, J. Am. Chem. Soc 130, 3420 (2008).

[26] J. Gibson, A. P. Monkman, and T. J. Penfold, ChemPhysChem 17, 2956 (2016).

[27] X. K. Chen, S. F. Zhang, J. X. Fan, and A. M. Ren, J. Phys. Chem. C 119, 9728 (2015).

[28] K. J. Borowski and R. E. Connors, J. Photochem. 16, 75 (1981).

[29] J. G. Langan, E. V Sitzmann, and K. B. Eisenthal, Chem. Phys. Lett. 124, 59 (1986).

[30] E. van Lenthe, A. Ehlers, and E. Baerends, J. Chem. Phys. 110, 8943 (1999).

[31] F. Wang and T. Ziegler, J. Chem. Phys. 123, 154102 (2005).

[32] E. Y. Li, T. Jiang, Y. Chi, P.-T. Chou, E. Yu-Tzu Li, T. Jiang, Y. Chi, and P.-T. Chou, Phys. Chem. Chem. Phys. 16, 26184 (2014).

[33] N. G. Martinelli, J. Idé, R. S. Sánchez-Carrera, V. Coropceanu, J. L. Brédas, L. Ducasse, F. Castet, J. Cornil, and D. Beljonne, J. Phys. Chem. C 114, 20678 (2010).

[34] J.-L. Brédas, D. Beljonne, V. Coropceanu, and J. Cornil, Chem. Rev. 104, 4971 (2004).

[35] P. K. Samanta, D. Kim, V. Coropceanu, and J.-L. Brédas, J. Am. Chem. Soc. 139, 4042 (2017).

[36] C. Baleizão and M. N. Berberan-Santos, J. Chem. Phys. 126, 204510 (2007).

[37] J. Yguerabide and M. Burton, J. Chem. Phys. 37, 1757 (1962).

[38] B. Yurash et al. (unpublished).

[39] T. Nakagawa, S.-Y. Ku, K.-T. Wong, and C. Adachi, Chem. Commun. 48, 9580 (2012). 